\title{
PREVALENCIA Y FACTORES ASOCIADOS A SINTOMATOLOGÍA DEPRESIVA EN ADULTOS MAYORES DEL CENTRO DE SALUD “MICAELA BASTIDAS" LIMA-PERÚ
}

\author{
PREVALENCE AND FACTORS ASSOCIATED WITH DEPRESSIVE SYMPTOMATOLOGY IN OLDER ADULTS OF THE \\ "MICAELA BASTIDAS" HEALTH CENTER, LIMA-PERU
}

Christian Ponce-Torres', Luigui Viteri-Condori', Pablo Ramos-Martínez', Wendy Nieto-Gutiérrez', Jesús Cueva-Velazco

\begin{abstract}
RESUMEN
Objetivo: Evaluar los factores asociados a la sintomatología depresiva en adultos mayores residentes del centro de salud asistentes al centro de salud "Micaela Bastidas" de Lima-Perú. Métodos: Estudio transversal analítico en adultos mayores de 60 años residentes de una zona urbano marginal de Lima. Se evaluó la variable dependiente usando la escala de depresión geriátrica de 15 ítems, se consideró la presencia de sintomatología depresiva con un puntaje $\geq 6$. Los factores asociados a la sintomatología depresiva, se calcularon usando razones de prevalencia crudas y ajustadas con intervalos de confianza al 95\% (IC 95\%) mediante regresiones de poisson con varianza robusta. Resultados: De los 142 adultos mayores (edad $70,4 \pm 8,14$ años), la prevalencia de la presencia de sintomatología depresiva fue $55,6 \%$ (mujeres: $39,6 \%$ y hombres; 53,4\%). El no tener escolaridad (RPa: 1,40; IC 95\%: 1,04 - 1,88) y no estar trabajando (RPa: 1,50; IC 95\%: 1,09 - 2,05) fueron factores asociados que aumentaban la prevalencia de sintomatología depresiva. Conclusión: La prevalencia de presencia de sintomatología depresiva en adultos mayores de una zona urbano marginal de Perú fue alta. Los adultos mayores que no completaron su escolaridad y no trabajaban presentaron más probabilidades de presentar sintomatología depresiva.
\end{abstract}

Palabras clave: Depresión; Anciano; Servicios preventivos de salud (fuente: DeCS BIREME).

\begin{abstract}
Objective: To evaluate the factors associated with depression in older adults in a marginal urban area of Peru. Methods: It is a cross-sectional analytical study in adults over 60 years of age in a marginal urban area of Lima. The dependent variable was evaluated using the 15 -items geriatric depression scale, considering presence of depression with a score $\geq 6$. The factors associated with depression were calculated using raw and adjusted prevalence ratios with $95 \%$ confidence intervals $(95 \% \mathrm{Cl})$ using poisson regressions with robust variance. Results: Of the 142 older adults (age: $70.4 \pm 8.14$ years), the prevalence of the presence of depression was 55.6\% (women: $39.6 \%$ and men: 53.4\%). The education (RPa: 1.40, 95\% Cl: $1.04-1.88$ ) and not currently working status (RPa: $1.50,95 \% \mathrm{Cl}: 1.09-2.05$ ) were associated factors that increased the prevalence of depression. Conclusion: The prevalence of depression in older adults in a marginal urban area of Peru was high. Older adults who did not complete their schooling and did not work were more likely to develop depression.
\end{abstract}

Key words: Depression; Frail elderly; Aged; Primary prevention (source: MeSH NLM). 


\section{INTRODUCCIÓN}

La depresión es uno de los trastornos neuropsiquiátricos más importantes en adultos mayores, y es una de las causas de discapacidad que disminuye la funcionalidad física, cognitiva y social, de esta manera afecta la calidad de vida y su bienestar ${ }^{(1)}$.

Para el año 2017, la Organización Mundial de Salud (OMS) estimó una prevalencia de depresión del 7\% en personas mayores de 60 años $^{(2)}$. Sin embargo, estudios han reportado cifras variables de depresión en adultos mayores que difiere según el contexto a nivel asistencial en pacientes de hospitalización: 32,8\% en Chengdu, China ${ }^{(3)} ; 44 \%$ en East Anglia, Reino Unido ${ }^{(4)} ; 47 \%$ en Belo Horizonte, Brasil(5); en consulta externa: $22 \%$ en Bikaner, India ${ }^{(6)} ; 29,9$ en Izmir, Turquía ${ }^{(7)}$; en centros de atención primaria de salud: $39,6 \%$ en Ethekwini, Sudafrica ${ }^{(8)}$; $45,3 \%$ en Kavre, Nepal(9); $47,2 \%$ en Beirut, Libano ${ }^{(10)}$; en adultos mayores de la comunidad: $27,8 \%$ en Sri Lanka, Singapur(11); $29,5 \%$ en Antiquia, Colombia ${ }^{(12)} ; 36 \%$ en Bangalore, India ${ }^{(13)} ; 52,6 \%$ en Vitoria da Conquista, Brasil ${ }^{(14)}$.

En cuanto a los factores asociados a la depresión geriátrica se ha reportado en la literatura con mayor frecuencia el ser mujer ${ }^{(8,15,16)}$, carecer de escolaridad ${ }^{(15,17)}$, el deterioro cognitivo ${ }^{(16,17)}$, vivir sin pareja ${ }^{(8,15)}$, padecer de problemas de salud ${ }^{(18,19)}$, hospitalizaciones previas ${ }^{(19)}$, falta de apoyo social(19,20), bajo nivel socioeconómico ${ }^{(16)}$, no trabajar ${ }^{(21)}$, entre otros.

Existen escalas que sirven como instrumentos de screening para evaluar la sintomatología depresiva en población geriátrica, entre ellos, Scale Geriatric Depression (GDS) creada por Yesavage, et al, en el año 1983, originalmente consta de 30 preguntas con respuestas dicotómicas de si o no ${ }^{(22)}$, dicha escala es frecuentemente usada en estudios epidemiológicos de depresión en adultos mayores. En nuestra búsqueda de estudios peruanos publicados sobre el tema que hayan utilizado el GDS, en su versión corta de 15 preguntas, se han reportado en su mayoría cifras altas de depresión: $51,6 \%$ (con puntaje de 5 o más) en un asentamiento humano del distrito de La Molina, Lima ${ }^{(23)}$; 8,4\% (con puntaje de 6 o más) en la comunidad del distrito de San Martin de Porres, Lima ${ }^{(24)} ; 48,2 \%$ (con puntaje 6 o más) en la comunidad del distrito de Breña, Lima ${ }^{(25)} ; 69,4 \%$ en la comunidad de Masma Chicche, Junin ${ }^{(26)} ; 64,9 \%$ en un hospital nacional de Ica, Ica ${ }^{(27)}$ en estos dos últimos estudios no se describe el punto de corte del GDS-15.

Existen pocos trabajos publicados sobre depresión en adultos mayores de las comunidades urbano marginales, considerando su situación de pobreza y el escaso acceso a servicios de atención en salud mental. Esto dificultaría la planificación de estrategias preventivas que mejoren la detección temprana de los síntomas depresivos y mantengan la funcionalidad en este grupo etario.

Por ello, el objetivo del estudio es determinar los factores asociados a la sintomatología depresiva en los adultos mayores residentes en una zona urbano marginal el "asentamiento humano Micaela Bastidas I" del distrito de Ate Vitarte en Lima, Perú.

\section{MÉTODOS}

\section{Diseño y lugar del estudio}

Se realizó un estudio observacional, analítico y transversal en adultos mayores de 60 años a más, del Asentamiento Humano Micaela Bastidas I, en Lima, durante el mes de abril a julio del 2014.

El "Asentamiento Humano Micaela Bastidas I" es una zona urbano marginal fundada en el año 1985 ubicado en el distrito de Ate Vitarte en Lima Este, sus habitantes son de estrato socioeconómico bajo. Esta localidad cuenta con un centro de atención primaria salud Micaela Bastidas nivel I-3 de la MicroRed Ate II del Ministerio de Salud.

\section{Población y muestra}

Los participantes fueron un total de 200 adultos mayores de 60 años en el año 2014 que se atendían en el Centro de Salud Micaela Bastidas, realizamos la encuesta a todos los adultos mayores. Entre los criterios de elegibilidad, se incluyeron a adultos mayores que eran atendidos en el centro de salud "Micaela Bastidas" durante el periodo del estudio, y que habían aceptado participar mediante un consentimiento informado firmado. Se excluyeron adultos mayores con algún grado de deterioro cognitivo que fueron previamente diagnosticados por un médico información proporcionada por el centro de salud como se puede observar en la Figura 1. Respondieron 146 adultos mayores, luego de un control de calidad se eliminaron 4 cuestionarios que estaban incompletos. La muestra final fueron de 142 participantes obteniendo una respuesta del $71 \%$ del total de adultos mayores.

\section{Procedimientos}

Los autores del estudio estuvieron a cargo de la recolección de datos mediante entrevistas a cada adulto mayor. Se solicitó permiso al centro de salud "Micaela Bastidas" para encuestar a los adultos mayores que acudían a sus citas al establecimiento. Además, cada entrevistador realizó visitas tres veces a la semana a las viviendas del "Asentamiento Humano Micaela Bastidas" en base a un plano de calles y manzanas de la zona, en cada vivienda se preguntó la presencia de un 
adulto mayor de 60 años. La participación en el estudio fue voluntaria, previamente se brindó información acerca de los objetivos del estudio y un consentimiento informado a los participantes.

\section{Variables}

La variable dependiente de interés fue sintomatología depresiva en adultos mayores. Para ello, se usó la escala GDS-15 de Yesavage et. al, ha sido probada y usada extensamente con múltiples poblaciones de adultos mayores a nivel mundial ${ }^{(22)}$. En el presente estudio se utilizó la versión traducida al español del Cuestionario GDS-15, contiene un total de 15 preguntas cada pregunta un vale 1 punto las respuestas son dicotómicas sí o no. De los 15 puntos, un puntaje mayor o igual 6 puntos indica presencia de síntomas depresivos ${ }^{(28)}$.

En cuanto a las variables independientes fueron seleccionadas a partir de estudios similares: sexo; edad (en terciles); situación conyugal se categorizó en aquellos con pareja (quienes estaban casados o convivientes) y sin pareja (solteros, viudos o divorciados); nivel educativo en sin escolaridad (sin estudios o primaria incompleta) y con escolaridad (primaria completa, secundaria o superior); procedencia (Lima u otras ciudades del Perú); problema de salud referido por el participante (si o no); y pertenecer a un club del adulto mayor (si o no).

\section{Análisis de datos}

La base de datos en Microsoft Excel 2010 fue exportada al software STATA en su versión 13. En el análisis descriptivo se usaron frecuencias absolutas y relativas.
Para evaluar los factores asociados con los síntomas depresivos se usó regresión de Poisson cruda y ajustada con varianza robusta y se calcularon las prevalencias de ratios (PR) y su intervalo de confianza al 95\%, considerando significancia estadística un $p<0,05$. Para los modelos ajustados se incluyeron las variables sexo, edad, nivel educativo, situación conyugal, procedencia, problema de salud y club del adulto mayor.

\section{Aspectos éticos}

En el presente estudio se cumplieron con los principios éticos de la Declaración de Helsinki. La participación en el estudio fue en todo momento voluntaria, se mantuvo la confidencialidad de los datos y no fueron sometidos a ningún otrotipo de riesgo por su participación, previamente se expuso a los participantes un consentimiento informado escrito y oral. Al final se entregaron los resultados al centro de salud Micaela bastidas.

\section{RESULTADOS}

En total 142 adultos mayores aceptaron participar en el estudio, el $67,6 \%$ fueron mujeres, la edad promedio y desviación estándar fue 70,4 $\pm 8,14$. El 43,7\% tenían situación conyugal de casados. La mayoría de los participantes $85,9 \%$ provenían de otras ciudades, el $69,0 \%$ manifestaron tener algún problema de salud y el $71,8 \%$ no pertenecían a un club del adulto mayor. Respecto al puntaje GDS-15, 44,4\% tuvieron entre 0 a 5 puntos (sin síntomas depresivos), 43,7\% entre 6 a 10 puntos (riesgo de sintomatología depresiva) y 12,0\% entre 11 a 15 puntos (sintomatología depresiva establecida) (Tabla 1).

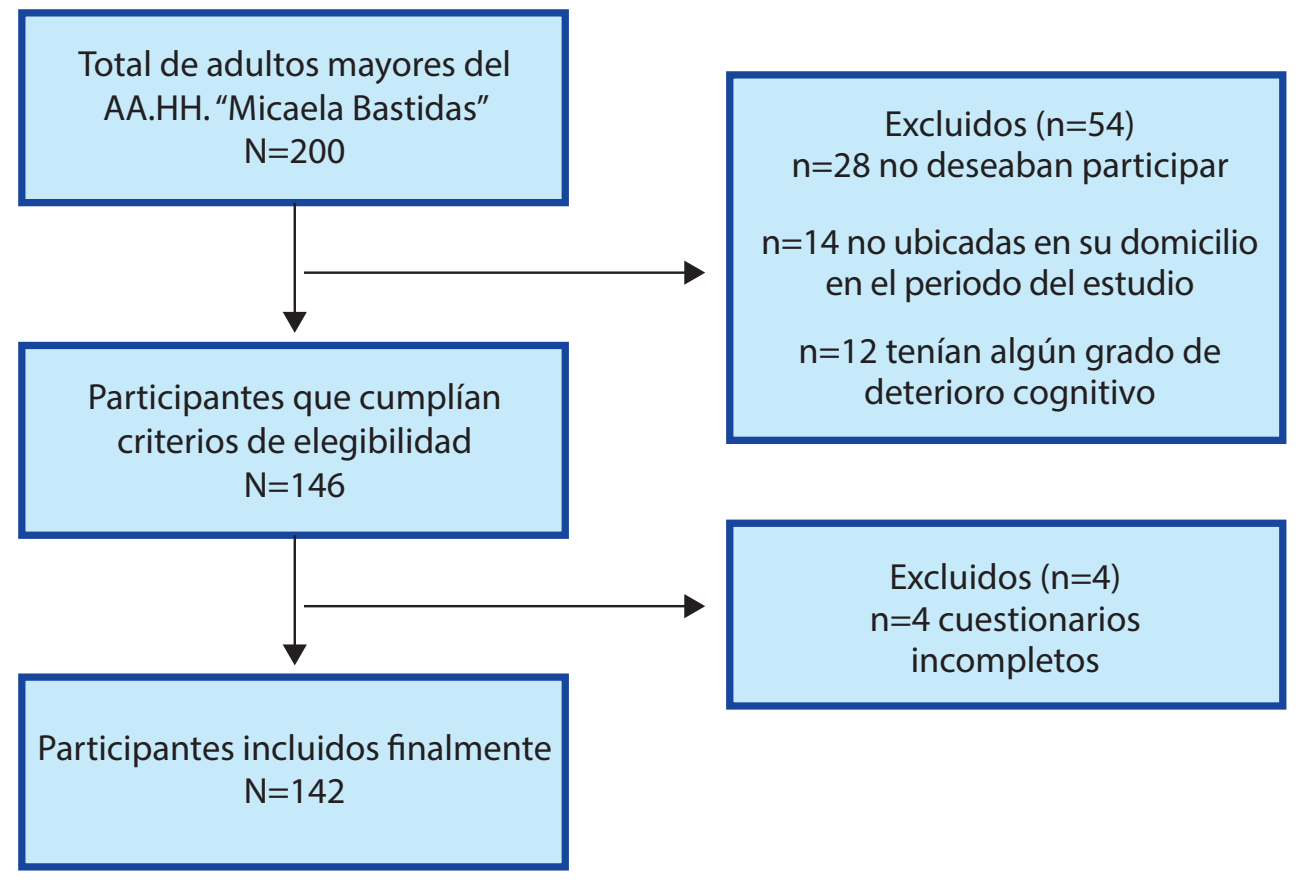

Figura 1. Diagrama de inclusión de participantes en el estudio. 
Tabla 1. Datos sociodemográficos y escala GDS-15 en la población estudiada ( $n=142)$.

\section{Variables}

\section{$\mathbf{N}(\%)$}

\section{Sexo}

Femenino

$96(67,6)$

Masculino

$46(32,4)$

\section{Situación conyugal}

Casado(a)

$62(43,7)$

Conviviente

$10(7,0)$

Divorciado(a)

Soltero(a)

Viudo(a)

\section{Nivel Educativo}

Analfabeto(a)

$28(19,7)$

Primaria incompleta

$38(26,8)$

Primaria completa

$28(19,7)$

Secundaria incompleta

Secundaria completa

$29(20,4)$

Superior

\section{Procedencia}

Lima

Provincia

\section{Problema de salud}

No

$\mathrm{Si}$

\section{Pertenece a un club del adulto mayor}

No

Si

\section{Situación laboral}

No trabaja

Si trabaja

$68(47,9)$

\section{Puntaje de GDS-15}

Normal

Riesgo de sintomatología depresiva

$62(43,7)$

Sintomatología depresiva

En cuanto a los factores asociados a tener presencia de síntomas depresivos (GDS-15 con un puntaje $\geq 6$ puntos), en el análisis ajustado se encontró que fueron las siguientes variables: no tener escolaridad $(R P=1,40$;
IC95\%:1,04 - 1,88) y no trabajar (RP:1,50; IC95\%:1,09 - 2,05). No se encontró asociación significativa con el sexo, edad, pareja, procedencia, problema de salud y pertenecer al club del adulto mayor (Tabla 2). 
Tabla 2. Factores asociados a la depresión en adultos mayores.

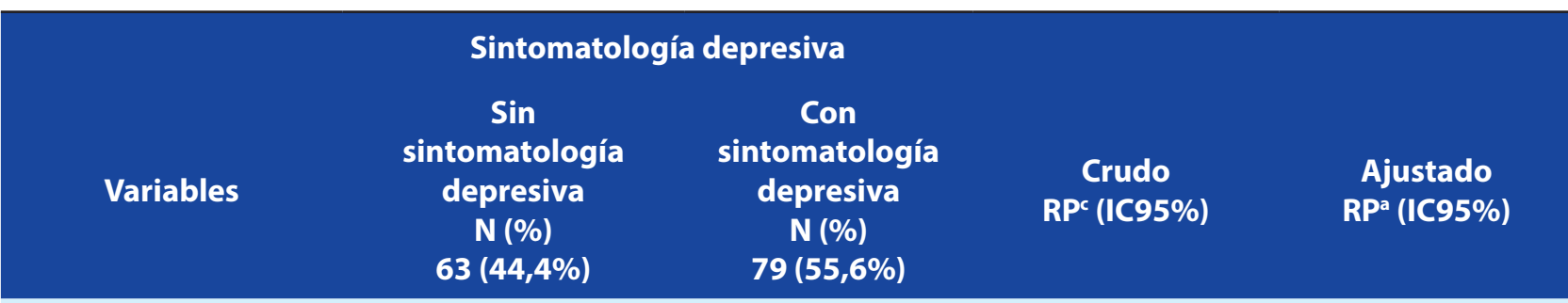

\section{Sexo}

Femenino

$38(39,6)$

$58(60,4)$

Ref

Ref

Masculino

$25(54,3)$

$21(45,7)$

$0,76(0,53-1,08)$

$0,87(0,63-1,21)$

\section{Edad}

60 a 68 años

$34(47,9)$

$37(52,1)$

Ref

Ref

69 a 90 años

$29(40,8)$

$42(59,2)$

$1,14(0,84-1,53)$

$0,99(0,73-1,33)$

\section{Situación conyugal*}

Con pareja

$36(50,0)$

$36(50,0)$

Ref

Ref

Sin pareja

$27(38,6)$

$43(61,4)$

$1,23(0,91-1,65)$

$1,23(0,92-1,64)$

\section{Nivel educativo}

Con escolaridad

$42(55,3)$

$34(44,7)$

Ref

Ref

Sin escolaridad

$21(31,8)$

$45(68,2)$

$1,52(1,13-2,06)$

$1,40(1,04-1,88)$

Procedencia

Lima

$12(60,0)$

$8(40,0)$

Ref

Ref

Provincia

$51(41,8)$

$71(58,2)$

$1,46(0,84-2,54)$

$1,35(0,85-2,16)$

\section{Problema de salud}

$\begin{array}{ll}\text { No presenta } & 24(54,5) \\ \text { Si presenta } & 39(39,8)\end{array}$

$20(45,5)$

Ref

Ref

Si presenta

$39(39,8)$

$59(60,2)$

$1,32(0,92-1,90)$

$1,18(0,82-1,69)$

\section{Pertenece al Club del Adulto Mayor}

\begin{tabular}{|c|c|c|c|c|}
\hline Si & $17(42,5)$ & $23(57,5)$ & Ref & Ref \\
\hline No & $46(45,1)$ & $56(54,9)$ & $0,95(0,69-1,32)$ & $1,10(0,79-1,52)$ \\
\hline \multicolumn{5}{|c|}{ Situación laboral } \\
\hline Sí trabaja & $39(57,4)$ & $29(42,6)$ & Ref & Ref \\
\hline No trabaja & $24(32,4)$ & $50(67,6)$ & $1,58(1,15-2,18)$ & $1,50(1,09-2,05)$ \\
\hline
\end{tabular}

*Estado conyugal con pareja (casados o convivientes) y sin pareja (solteros, viudos o divorciados).

†Nivel de educativo con escolaridad (sin estudios o primaria incompleta) y sin escolaridad (primaria completa, secundaria y superior.

\section{DISCUSIÓN}

\section{Porcentajes de depresión}

Del total 142 adultos mayores, el 55,6\% tuvieron mayor prevalencia de sintomatología depresiva de acuerdo al GDS-15. Cifra alta comparado con otros estudios que utilizaron el mismo instrumento y punto de corte (puntaje mayor o igual a 6): 48,1\% en Patras y Trípolis, Grecia ${ }^{(29)} ; 45,4 \%$ en Chihuahua, México ${ }^{(30)} ; 43 \%$ en Asunción, Paraguay ${ }^{(31)} ; 31,1 \%$ en Jequié, Brasil ${ }^{(32)}$; 29,5\% en Antioquia, Colombia ${ }^{(12)} ; 27,8 \%$ en Sri Lanka, Singapur ${ }^{(11)} ; 19,7 \%$ en Guadalajara, España ${ }^{(18)}$.
En Perú estudios publicados han reportado, en el 2010 en un asentamiento humano "Viña Alta" del distrito de la Molina de Lima en 112 ancianos encontraron que el $51,6 \%$ tenían depresión con un puntaje de 5 o más ${ }^{(24)}$. En el 2013, un estudio en 369 adultos mayores del distrito de Breña de Lima, 48,2\% tenían depresión con puntaje 6 o más ${ }^{(23)}$. En el 2012, un estudio realizado en 72 personas de una comunidad de Masma Chicche en Junín, 69,4\% presentaron depresión no describe el puntaje usado en el GDS-15(26). Estos porcentajes son ligeramente altos comparado con nuestro estudio, probablemente tuvieron menos oportunidades de ser atendidas por 
médicos especialistas (psiquiatría, medicina interna, geriatría, etc), sumado a la no concientización de este síndrome geriátrico en adultos mayores y sus familiares. Lo anterior explicaría, la limitada utilización de servicios de salud mental en ancianos de zonas urbano marginales por la falta de movilidad, transporte, distancia, tiempo de viaje y gastos, ya que es común que se ubiquen en la periferia de la ciudad.

\section{Factores asociados}

\section{Pareja}

Si bien encontramos que la tercera parte de los adultos mayores sin pareja (solteros, viudos o divorciados) tuvieron riesgo de sintomatología de depresión, no se halló significancia estadística. El no tener pareja, si fue un factor asociado significativamente en adultos mayores de Pakistán ${ }^{(33)}$, India ${ }^{(34)}$ y Colombia ${ }^{(35)}$, esto se podría deber a los cambios somáticos y emocionales que experimentan los adultos mayores sin pareja, con mayor tendencia a presentar episodios depresivos en el envejecimiento. La ausencia de significancia estadística en nuestro estudio se debería a limitaciones en el tamaño muestral de la población de estudio, se recomienda realizar más estudios que evalúen este tema.

\section{Escolaridad}

Encontramos que los adultos mayores que no completaron su escolaridad estuvieron asociados con tener riesgo de sintomatología depresiva, este resultado es similar a lo reportado por estudios previos en adultos mayores de Sudáfrica ${ }^{(8)}$, Colombia ${ }^{(12)}$, Brasil $^{(17)}$, Nepal(20) y México(20). El nivel de educativo puede influir en la calidad de vida del adulto mayor ya que forma parte de las experiencias de aprendizaje y reserva cognitiva, de manera que una persona con un mayor nivel educativo podría ser un coadyuvante para afrontar situaciones estresantes evitando que alteren su estado emocional por ende disminuyendo el riesgo de depresión ${ }^{(36)}$. Asimismo, se deberían implementar estrategias encaminadas a mejorar el nivel educativo en adultos mayores de zonas urbano marginales.

\section{Trabajar}

En nuestro estudio los adultos mayores que no trabajaban tuvieron mayor prevalencia de depresión. Este hallazgo también fue encontrado en estudios realizados en Pakistán ${ }^{(21)}$ y India ${ }^{(37)}$. La población que estudiamos era pobre, es probable que los adultos mayores que no tenía alguna actividad laboral vivan una situación económica más tensa y estresante en sus hogares con mayor probabilidad de sufrir depresión.

\section{Limitaciones}

Entre las limitaciones del presente estudio, el escaso tamaño de muestral es posible que en el periodo de la recolección no se hayan incluido a todos los adultos mayores puesto que no existía un registro real del total de adultos mayores en lugar de estudio. La población encuestada se limitó solo a una zona urbanomarginal de la ciudad de Lima, entonces los resultados no pueden ser extrapolados a otras zonas del país. Nuestros resultados permiten dar una perspectiva de lo que podría estar sucediendo en contextos como en comunidades urbanomarginales. El uso del cuestionario GDS-15 como tamizaje, no permite dar un diagnóstico definitivo de depresión. Sin embargo, es un instrumento corto, compresible y ya ha sido usado en estudios similares en Perú.

\section{CONCLUSIÓN}

En conclusión, encontramos que cinco de cada diez adultos mayores tuvieron prevalencia de síntomas depresivos. La prevalencia de depresión fue alta en aquellos adultos mayores que no completaron su escolaridad y no trabajaban. Los hallazgos del estudio llaman a la reflexión a realizar más estudios de salud mental en adultos mayores que residen zonas urbano marginales, y al personal de salud de la importancia de diagnosticar y tratar la depresión en adultos mayores que buscan alivio para sus síntomas. Asimismo, la planificación de estrategias que potencien la atención geriátrica en los establecimientos primaria de salud.

Contribuciones de autoría: Los autores participaron en la génesis de la idea, diseño de proyecto, recolección e interpretación de datos, análisis de resultados y preparación del manuscrito del presente trabajo de investigación.

Financiamiento: Autofinanciado.

Conflicto de interés: Los autores declaran no tener conflicto de interés en la publicación de este artículo.

Recibido: 25 de febrero 2020

Aprobado: 19 de marzo 2020

Correspondencia: Christian Ponce Torres.

Dirección: Av. El corregidor 1531, Las Viñas, La Molina, Lima-Perú.

Teléfono: +51995473883

Correo: christianfpt01@gmail.com 


\section{REFERENCIAS BIBLIOGRÁFICAS}

1. Copeland JR, Beekman AT, Braam AW, Dewey ME, Delespaul P, Fuhrer R, et al. Depression among older people in Europe: the EURODEP studies. World Psychiatry. 2004;3(1):45. Disponible en: https://www.ncbi.nlm.nih.gov/pmc/ articles/PMC1414664/pdf/wpa010045.pdf

2. Organizacion Mundial de Salud. La salud mental y los adultos mayores. Ginebra:OMS; 2017. Disponible en: http://www.who.int/es/news-room/factsheets/detail/la-salud-mental-y-los-adultos-mayores

3. Zou C, Chen S, Shen J, Zheng X, Wang L, Guan L, et al. Prevalence and associated factors of depressive symptoms among elderly inpatients of a Chinese tertiary hospital.Clin Interv Aging. 2018; 13: 1755-1762. DOI: 10.2147/ CIA.S170346

4. Cullum S, Tucker S, Todd C, Brayne C. Screening for depression in older medical inpatients. Int J Geriatr Psychiatry. 2006;21(5):469-76. DOI: 10.1002/ gps.1497

5. Serra MA, do Nascimento IMT, de Lima Guimarães G, da Silva SM, Goveia VR Mendoza IY. Prevalencia de síntomas depresivos en ancianos hospitalizados: un estudio comparativo. Rev enferm UERJ. 2019; 27: e36091. DOI: http://scielo. sld.cu/pdf/mgi/v33n4/a01_296.pdf

6. Prakash O, Gupta LN, Singh VB, Nagarajarao G. Applicability of 15-item Geriatric Depression Scale to detect depression in elderly medical outpatients. Asian J Psychiatr. 2009;2(2):63-5. DOI: 10.1016/j.ajp.2009.04.005

7. Durmaz B, Soysal $P$, Ellidokuz $H$, Turan Isik A. Validity and reliability of geriatric depression scale-15 (short form) in Turkish older adults. North Clin Istanb. 2018; 5(3): 216-220. DOI: 10.14744/nci.2017.85047

8. Padayachey U, Ramlall S, Chipps J. Depression in older adults: prevalence and risk factors in a primary health care sample. South African Family Practice. 2017;59(2):61-6. DOI: 10.1080/20786190.2016.1272250

9. Risal A, Giri E, Shrestha O, Manandhar S, Kunwar D, Amatya R, et al. Nepal Version of Geriatric Depression Scale-15 - A Reliability and Validation Study. J Nepal Health Res Counc. 2020;17(4):506-511. DOI: 10.33314/jnhrc.v17i4.1984

10. Chaaya M, Sibai AM, Roueiheb ZE, Chemaitelly H, Chahine LM, Al-Amin H,et al. Validation of the Arabic version of the short Geriatric Depression Scale (GDS 15). Int Psychogeriatr. 2008;20(3):571-81. DOI: 10.1017/S1041610208006741

11. Malhotra R, Chan A, Østbye T. Prevalence and correlates of clinically significant depressive symptoms among elderly people in Sri Lanka: findings from a national survey. International Psychogeriatrics. 2010;22(2):227-36. DOI: $10.1017 /$ S1041610209990871

12. Segura-Cardona A, Cardona-Arango D, Segura-Cardona Á, Garzón-Duque M. Riesgo de depresión y factores asociados en adultos mayores. Antioquia Colombia. 2012. Revista de Salud Pública. 2015;17:184-94. DOI: 10.15446/rsap. v17n2.41295

13. Sanjay T, Jahnavi R, Gangaboraiah B, Lakshmi P, Jayanthi S. Prevalence and factors influencing depression among elderly living in the urban poo locality of Bengaluru city. International Journal of Health \& Allied Sciences. 2014;3(2):105. DOI: 10.4103/2278-344X.132695.

14. Matias AGC, Fonsêca MdA, Gomes MdLdF, Matos MAA. Indicators of depression in elderly and different screening methods. Einstein (São Paulo). 2016;14(1):6-11. DOI: 10.1590/S1679-45082016AO3447.

15. Martina M, Ara MA, Gutiérrez C, Nolberto V, Piscoya J, editors. Depresión y factores asociados en la población peruana adulta mayor según la ENDES 2014-2015. Anales de la Facultad de Medicina; 2017: UNMSM. Facultad de Medicina. DOI: 10.15381 /anales.v78i4.14259.

16. Gibson R, Neita S, Abel W, James K, Eldemire-Shearer D. Sociodemographic factors associated with depressive symptoms among elderly persons from two communities in Kingston, Jamaica. West Indian Medical Journal. 2013;62(7):615-9. DOI: 10.7727/wimj.2012.273

17. Borges LJ, Benedetti TRB, Xavier AJ, d'Orsi E. Associated factors of depressive symptoms in the elderly: EpiFloripa study. Revista de saude publica. 2013;47(4):701-10. DOI: 10.1590/S0034-8910.2013047003844.

18. Urbina Torija JR, Flores Mayor JM, García Salazar MP, Torres Buisán L, Torrubias Fernández RM. Síntomas depresivos en personas mayores: Prevalencia y factores asociados. Gaceta Sanitaria. 2007;21(1):37-42. DOI: http://scielo.isciii. es/scielo.php?script=sci_arttext\&pid=S0213-91112007000100008

19. Santiago LM, Mattos IE. Depressive symptoms in institutionalized olde adults. Revista de saude publica. 2014:48:216-24. DOI: 10.1590/S0034 8910.2014048004965

20. Timalsina R, Sherpa P, Dhakal D. Factors associated with depression among elderly living in old age homes in Kathmandu Valley. Journal of institute of medicine. 2014;37(1). DOI: 10.3126/jpan.v4i1.16742
21. Taqui AM, Itrat A, Qidwai W, Qadri Z. Depression in the elderly: does family system play a role? A cross-sectional study. BMC Psychiatry. 2007: 25;7:57. DOI: 10.1186/1471-244X-7-57.

22. Yesavage JA, BrinkTL, Rose TL, Lum O, Huang V, Adey M, et al. Development and validation of a geriatric depression screening scale: a preliminary report. Journal of psychiatric research. 1983;17(1):37-49. DOI: 10.1016/00223956(82)90033-4

23. Juárez J, León $A$, Linares VA. Evaluación del grado de depresión de adultos mayores de 60 años del AA. HH "Viña alta"-La Molina, Lima-Perú. Horizonte Médico. 2012;12(2):28-31. Disponible en: http://www.horizontemedico.usmp. edu.pe/index.php/horizontemed/article/view/101

24. Silva-Fhon JR, Del Río-Suarez AD, Motta-Herrera SN, Coelho FabricioWehbe SC, Partezani-Rodrigues RA. Violencia intrafamiliar en el adulto mayor que vive en el distrito de Breña, Perú. Rev. Fac. Med. 2015;63(3):367 375. Disponible en: http://www.scielo.org.co/scielo.php?script=sci abstract\&pid=S0120-00112015000300004

25. Varela-Pinedo $L$, Chávez-Jimeno $H$, Tello-Rodríguez T, Ortiz-Saavedra $P$ Gálvez-Cano M, Casas-Vasquez P, et al. Perfil clínico, funcional y sociofamiliar del adulto mayor de la comunidad en un distrito de Lima, Perú. Revista Peruana de Medicina Experimental y Salud Pública. 2015;32(4):70916. Disponible en: http://www.scielo.org.pe/scielo.php?script=sci arttext\&pid=S1726-46342015000400012

26. Contreras AL, Mayo GVA, Romaní DA, Silvana Tejada G, Yeh M, Ortiz PJ, et al. Malnutrición del adulto mayor y factores asociados en el distrito de Masma Chicche, Junín, Perú. Revista Medica Herediana. 2013;24(3):186-91. Disponible en: $\quad$ http://www.scielo.org.pe/scielo.php?script=sci arttext\&pid=S1018$130 \times 2013000300002$

27. Consuelo De La Cruz-Mitac CY, Quispe-Ilanzo MP, Oyola-García AE, Portugal-Medrano MA, Lizarzaburu-Córdova EE, Rodríguez-Chacaltana FW. Depresión en adultos mayores con enfermedades crónicas en un hospital general del Perú. Rev cuerpo méd HNAAA. 2017;10(4):205-211. DOI: 10.35434/ rcmhnaaa.2017.104.18.

28. Orcos RO, Fort MS, Khajoui AK, Aparicio SV, Del Valle RDD. Validación de la versión española de 5 y 15 ítems de la Escala de Depresión Geriátrica en personas mayores en Atención Primaria. Revista clinica española. 2007 207(11): 559-562. DOI: 10.1016/S0014-2565(07)73477-X

29. Argyropoulos K, Bartsokas C, Argyropoulou A, Gourzis P, Jelastopulu E. Depressive symptoms in late life in urban and semi-urban areas of South-West Greece: An undetected disorder?. Indian J Psychiatry. 2015; 57(3): 295-300.

30. Flores-Padilla L, Ramírez-Martínez FR, Trejo-Franco J. Depresión en adultos mayores (AM) con pobreza extrema pertenecientes a un Programa Social en Ciudad Juárez, Chihuahua, México. Gac Med Mex. 2016;152:439-43. DOI: 10.4103/0019-5545.166617

31. Piñánez García MC, Re Domínguez ML. Perfil clínico, epidemiológico y valoración geriátrica funcional en el barrio San Miguel de Asunción, Paraguay. Rev Virtual Soc Parag Med Int. 2015;.2 (2): 63-69. Disponible en: https://dialnet. unirioja.es/servlet/articulo?codigo $=5678110$

32. Dos Santos Ribeiro V, Souza Rosa R, Cruz Sanches GJ, Santos Ribeiro IJ, Augusto Cassotti C. Calidad de vida y depresión en idosos en el contexto domiciliar. Enferm Actual Costa Rica. 2018; 4(34):1-14. Disponible en: https:// revistas.ucr.ac.cr/index.php/enfermeria/article/view/30983

33. Javed S. Role of Sociocultural Factors in Depression among Elderly of Twin Cities (Rawalpindi and Islamabad) of Pakistan. Curr Gerontol Geriatr Res. 2014 2014: 230737. DOI: 10.1155/2014/230737

34. Akhtar H, Khan AM, Vaidhyanathan KV, Chhabra P, Kannan AT. Sociodemographic Predictors of Depression among the Elderly Patients Attending Out Patient Departments of a Tertiary Hospital in North India. Int J Prev Med. 2013;4(8):971-5. Disponible en: https://www.ncbi.nlm.nih.gov/pmc/articles/ PMC3775177/

35. Borda Pérez M, Anaya Torres MP, Pertuz Rebolledo MM, Romero de León L, Suárez García A, Suárez García A. Depresión en adultos mayores de cuatro hogares geriátricos de Barranquilla (Colombia): prevalencia y factores de riesgo. Salud, Barranquilla. $2013 ; 29(1$ ): 64-73. Disponible en: http:// rcientificas.uninorte.edu.co/index.php/salud/article/viewArticle/3397

36. Giacoman C, Funes D, Guzmán L, Ramos TM. Depresión y escolaridad en adultos mayores. Diálogos sobre educación. 2015; 2(2):1-11. Disponible en:http://dialogossobreeducacion.cucsh.udg.mx/index.php/DSE/article/ view/380

37. Thirthahalli C, Suryanarayana SP, Sukumar GM, Bharath S, Rao GN, Murthy NS. Proportion and factors associated with depressive symptoms among elderly in an urban slum in Bangalore. J Urban Health. 2014:91(6):1065-75. DOI: 10.1007/s11524-014-9903-6. 